\title{
¿HABLANDO CON MIRLOS? EL USO DE LA PERSONIFICACIÓN DE LOS ANIMALES EN LA LEYENDA ARTÚRICA

\section{Resumen}

Aunque los textos que conforman la literatura artúrica no suelen presentar rasgos fabulescos, sino, más bien, mitológicos, algunas narraciones artúricas usan características propias de las fábulas para desarrollar sus ideas moralistas. Este artículo se centra en el estudio de un texto medieval galés y uno contemporáneo en inglés para demostrar cómo se hace uso y aplican las técnicas de la fábula a la leyenda artúrica.

Palabras clave: Literatura artúrica, fábula, mitología, Mabinogion, animales.

\begin{abstract}
Even when Arthurian literature does not usually present characteristics of fables but, rather, mythological qualities, some texts make use of fable features in order to develop a moralistic viewpoint. This article deals with the study of a Welsh medieval text and a contemporary one in English to show how the technique of the fable is used and applied to the Arthurian legend.
\end{abstract}

Key words: Arthurian literature, fable, mythology, Mabinogion, animals.

\section{INTRODUCCIÓN}

De acuerdo con el prestigioso Dictionary of Literary Terms and Literary Theory, publicado por Penguin, se define fábula como una historia breve que indica una enseñanza moral para la que se utilizan criaturas no humanas o seres inanimados y, a veces, hombres y hasta dioses. Lo importante es el carácter moral que la fábula contiene. Kurt Spang ha defendido la idea de que las similitudes entre fábula y otros tipos de texto, como el cuento o el mito, han dado lugar a la desaparición de los límites entre estos.

La literatura artúrica, tanto medieval como contemporánea, ha hecho pocas veces uso de características típicas de las fábulas, como las mencionadas anteriormente, para desarrollar sus tramas, ideas e historias. Este artículo pretende centrarse en una serie de textos, tanto medievales como contemporáneos, para demostrar cómo los autores hacen uso de ciertas técnicas fabulescas en sus historias y veremos cómo, curiosamente, el texto contemporáneo que vamos a tratar tiene más de fábula que los medievales.

Kurt Spang defiende a la fábula como "uno de los géneros narrativos más antiguos y difundidos" (Spang, 2000: 112). Pese a su antigüedad, hay que resaltar que las características principales de la fábula parecen inalterables, ya que narran acontecimientos del pasado entre figuras estereotípicas e invariables la mayoría de las veces. La resolución al problema presentado suele ser rápida y, como se acaba

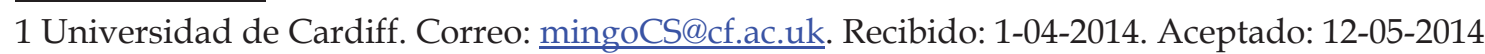


de apuntar, los personajes suelen ser animales. El problema que se plantea suele ser generalizable, esto es, aplicable universalmente, mientras que la actitud ante el mismo es crítica, satírica y didáctica. Los temas principales suelen centrarse en el enfrentamiento entre el fuerte y el débil y suelen castigarse los vicios, tales como la vanidad, el despotismo o todo comportamiento antinatural. Spang también añade que el hecho de la proximidad entre la fábula por un lado y la alegoría, la parábola o el cuento, por otro, ha originado la desaparición de límites entre estos subgéneros a la vez que ha permitido su adaptabilidad para "amoldarse a entornos y problemáticas diversas" (Spang, 2000: 114).

Es interesante resaltar aquí que en la lengua inglesa, aunque se usaban derivados de la palabra myth desde la Edad Media, esta misma palabra no se comenzó a usar hasta la década de los sesenta del siglo XVIII. La idea es que 'mythology' se entendía como una ciencia. Tal y como lo explica von Hendy, la idea de 'mythology' era

"not a body of stories, but the scholarly science of allegorical reading; "to mythologize" means, not to invent or relay a mythos, but to engage in that kind of interpretative practice." (von Hendy, 2002: 2)

El mismo autor confirma que la palabra fable, derivada de la latina fabula, se utilizó hasta entonces con el significado de mito.

Carlos Reis en su Diccionario de Narratología (2002) subraya que algunos textos modernos, caso que él ejemplifica con una colección del portugués Miguel Torga, no pueden considerarse fábulas pese a la presencia de animales y porque su estructura sigue, más bien, la de un cuento. No obstante, podemos asegurar que algunos rasgos de fábula subyacen, no solo en los textos medievales galeses que vamos a tratar, sino, sobre todo, en los contemporáneos.

Los animales en la literatura artúrica tienen un papel primordial, no solo a la hora de ayudar al protagonista a lograr su objetivo, sino también por su enseñanza moral en algunos casos y porque, en varios textos, aparecen en sueños premonitorios. Tal y como Paxon apunta,

"[i]n the form of the beast fable, the apologue seems to prescribe a simple relationship between the personifiers [intelligent entities that speak -these are the prosopa] and the personifieds [the naturally aphasic and non-intelligent animals]" (Paxon, 1994: 47).

El uso de los animales le ayuda al autor a criticar la sociedad:

Often, animal apologues will present an entire society, identical to that of humans, but comprised of animals at the individual members (...). Their societies are often specular images of Western human civilization, a point that has always made the animal apologue an especially appropriate vehicle for satire (Paxton, 1994: 47).

Veremos cómo esto se aplicará muy especialmente en el texto de T.H. White. 


\section{LOS ANIMALES EN LOS MABINOGION}

El primero de los textos que vamos a tratar es el de los Mabinogion, colección de once relatos que, aunque más antiguos en origen, se recogieron en el siglo XI en dos manuscritos (el MS Peniarth 4-5 en el Llyfr Gwyn Rhydderch, en Aberystwyth, y el MS Jesus College 111 del Llyfr Coch Hergest en Oxford). No todas las once historias son de temática artúrica, ya que algunas son historias de dioses celtas que sufren, viven y padecen las mismas pasiones que los mortales. La más famosa de las historias de los Mabinogion se llama "Culhwch ac Olwen". En esta historia, la madrastra de Culhwch le impone una maldición o geis tras rechazar el amor de su hija: no podrá casarse con otra mujer que no sea Olwen, la hija del temible gigante Ysbaddaden, hasta que lo haya matado. Al oír el nombre de la joven, inmediatamente Culhwch se enamora de ella y decide ir a la corte de su primo, el rey Arturo, para pedirle ayuda. Culhwch invoca su ayuda mencionando a más de doscientas personas de la corte. Esta lista, junto con el diálogo de Culhwch y Ysbaddaden y la búsqueda de Mabon, dios celta que desapareció tres días después de su nacimiento y que nadie sabe dónde está, forman los tres ejes de la historia y es importante que prestemos especial atención a este último ya que para la liberación de Mabon, los hombres de Arturo necesitarán la ayuda de una serie de animales. Estos tienen la capacidad de comunicarse con uno de los hombres del rey, Gwrhyr Gwalstawd lethoedd (que significa Gwrhyr, intérprete de lenguas), ya que, como Arturo le dice: "You know all the languages, and can speak the same language as some of the birds and the beasts" (Davies, 2008: 203). Así, los hombres de Arturo llegan hasta donde está el primero de los animales, el mirlo de Cilgwri, que les confiesa que no sabe dónde está Mabon, pero que los llevará hasta el segundo animal, el ciervo de Rhedynfre, ya que, tal y como les dice el mirlo, es más viejo que él. El ciervo, por su parte, les comenta que aunque lleva mucho tiempo viviendo en la zona, no ha oído nunca hablar de Mabon, pero se ofrece a llevarlos hasta otro animal que es aún más viejo que él: el búho de Cwm Cawlwyd. Con este corren la misma suerte y el búho les comenta: “I shall be a guide to Arthur's messengers until you come to the oldest animal in this world, and the one who has wandered mostthe Eagle of Gwernabwy" (Davies, 2008: 204). Sin embargo, con el águila vuelven a encontrarse con el mismo problema: no ha oído hablar de Mabon, pero les dice que conoce a un animal aún más viejo que ella, el salmón de Llyn Lliw, que los lleva a lomos suyos hasta Gloucester, donde Mabon está encarcelado y al que Arturo y sus hombres finalmente liberan.

El motivo literario del "animal más viejo del mundo", que se desarrolla en esta parte del cuento, tiene paralelos en las literaturas hindú y persa y la tradición galesa también hace uso del tema en otro de sus textos medievales, las Triads, donde no aparece el salmón de Llyn Lliw, pero sí el mirlo, el búho y el águila. Algunas de las personas, 
por otra parte, que se nombran en varios de los Mabinogion son personas a las que las han convertido en animales, otro motivo típico de la literatura celta. Algunos de esos animales, conservan aún algunas características humanas mientras que otros parecen haber perdido toda condición humana, como es el caso de la perra Rhymi, que atormenta a los habitantes del área de Aber Dugleddyf y mata a su ganado junto con sus dos cachorros. Pero cuando Arturo va a darles caza, "God changed them back into their own shape for Arthur" (Davies, 2008: 205). La caza del jabalí Twrch Trwyth ocupa buena parte de las aventuras que el gigante Ysbaddaden les prepara a Culhwch y a sus hombres. Twrch es un fiero jabalí que, según se nos informa, "he laid waste a fifth of Ireland (...) The third day Arthur himself fought against him, for nine nights and nine days. He only killed a single piglet" (Davies, 2008: 209). Twrch, de nuevo, es un ser humano al que Dios ha dado la forma de jabalí para castigarle por sus pecados. Gwrhyr, el intérprete, que toma forma de pájaro para hablar con él, le pide que vaya a hablar con Arturo, a lo que uno de los hijos del jabalí responde:

By Him who shaped us in this image, we will not do and we will not say anything to help Arthur. God has done us enough harm by shaping us in this image, without you too coming to fight us (Davies, 2008: 209).

Gwrhyr le comenta que, entonces, Arturo luchará contra ellos para conseguir el peine y la navaja escondidos tras las orejas de Twrch y que servirán para preparar al gigante Ysbaddaden para la boda de su hija, pero Grugyn Gwyrch Eraint, el jabato, le reta: "tomorrow morning we will set off from here, and we will go to Arthur's land, and there will be wreak and the greatest havoc possible" (Davies, 2008: 210).

Este episodio de la caza de Twrch Trwyth puede leerse de tres formas diferentes que, no obstante, no se contraponen entre sí. Por un lado, puede leerse literalmente, como una aventura que los hombres de Arturo realizan para ayudar a uno de los suyos y su consecución equivale no solo a un triunfo, sino a la mejora de esos hombres como guerreros. Por otro lado, tiene un valor cultural, ya que refleja uno de los pasatiempos más populares de la sociedad celta a lo largo de la Edad Media: la caza del jabalí, que en algunos textos representaba (y aún representa en textos artúricos contemporáneos) un rito de pasaje hacia la madurez. Y, por último, puede leerse como un texto histórico, ya que el camino que toma Twrch en su ataque de las tierras de Gales, profetizado por su hijo, es el mismo que hicieron los invasores irlandeses de los siglos V y VI. Los invasores entraron por lo que hoy en día es la ciudad de St. David's en Pembrokeshire, siguieron por la costa sur de Gales hasta la región de Glamorgan y de ahí pasaron hacia el interior de Brycheiniog (Brecon actualmente). Todos estos puntos estaban conectados por una antigua calzada romana al lado de la cual se erigieron unas piedras con inscripciones en ogham, el alfabeto que usaban los irlandeses entonces.

En "Culhwch ac Olwen" se representa uno de los pilares básicos de la sociedad 
celta, la familia, así como uno de los mayores problemas de la misma: el temor a morir sin hijos. El maleficio que la madrastra impone a Culhwch es el trasfondo de ese temor. Pero Culhwch gana (y sobreentendemos que tendrá descendencia) gracias a la familia, que en galés medieval se denominaba de dos formas: cenedl era la familia de sangre (en la ley galesa todos los miembros que tuvieran un bisabuelo común) y teulu era la familia adquirida para la defensa de un señor, su mujer y sus hijos. Así, el jefe de una cenedl podría aceptar a otros miembros que le ayudarían a defender su descendencia en caso de guerra o batalla. Arturo y Culhwch son cenedl, mientras que la teulu de Arturo ayuda a Culhwch y, por consiguiente, pasa a ser también su propia teulu. Esta simbiosis de cenedl y teulu se observa especialmente en tres de los episodios que forman la historia de "Culhwch ac Olwen": la búsqueda de Olwen, la de Mabon y la caza de Twrch Trywth. En las tres, además, se observa que una serie de animales ha formado parte más o menos activa de la aventura y ha ayudado a la teulu a lograr su objetivo, lo que simboliza para algunos autores (Knight, 1983, entre ellos) una unión entre un clan guerrero y la naturaleza (en contra de un ser antinatural, como sería Ysbaddaden, o un humano convertido en jabalí). De hecho, la teulu de Arturo completará con éxito algunas de las tareas que el gigante les impone pero no lo hacen de la forma que se les asigna: por ejemplo, matan a otro jabalí (Ysgithrwyn) con el perro de caza de Arturo (Cafall), pero no con los perros que Ysbaddaden les había encomendado. El motivo antinatural del humano convertido en animal, del que hemos visto dos ejemplos en Twrch Trwyth y sus jabatos y en la perra Rhymi y sus cachorros, no es poco común en la literatura galesa, ya que en otro de los cuentos de los Mabinogion, "Math ap Mathwonwy", el protagonista homónimo de la historia, rey de Gwynedd, convierte en una serie de animales a sus dos sobrinos por haber violado a la doncella que lo ayuda a él. Así, durante el primer año, los convierte en ciervo y cierva y al año vuelven al castillo con un cervatillo. Math se queda con el cervatillo y a sus sobrinos los convierte en jabalí y puerca y vuelven al año con un jabato, con el cual se queda de nuevo Math antes de convertirlos en lobo y loba. Al año vuelven con un lobezno. Esta vez, Math los devuelve a su forma humana y a los tres hijos que han tenido, también los hace humanos y les da los nombres de Hyddwn, Hychddwn y Bleiddwn. La primera parte de los nombres son las de las palabras ciervo, cerdo y lobo que en la tradición celta se relacionaban con las características de un guerrero: majestad, fiereza y astucia. La transformación de personas en animales no es, sin embargo, una característica única de la literatura artúrica galesa: en el romance castellano "Tres hijuelos había el rey", este transforma a sus tres hijos en ciervo, perro y al último en moro, en lo que se ve una referencia antiislámica clara. 


\section{OTROS RASGOS ANIMALESCOS EN LA LITERATURA MEDIEVAL}

Antes de pasar a The Sword in the Stone (1938), primera obra en la pentalogía de T.H. White The Once and Future King, sería interesante destacar aquí una descripción "animalizada" de uno de los caballeros de la Mesa Redonda: Ywain. En la literatura medieval artúrica es conocido el episodio de la locura del caballero que se refugiaba como un ermitaño en un bosque hasta que volvía a recobrar la cordura y regresaba a la civilización, representada en Camelot. Sin embargo, en el largo romance medieval inglés Ywain and Gawain (siglo XV), la representación de esta locura da un paso más. Ywain, acusado por su mujer de haberla olvidado y de ocuparse más de sus deberes como caballero que de esposo, se vuelve loco y se encamina al bosque donde encuentra asilo y allí:

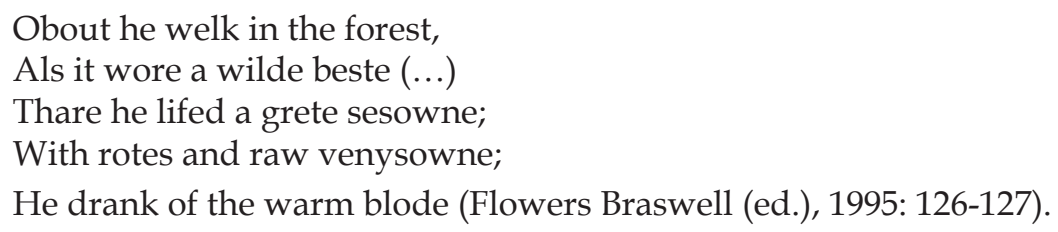

Obout he welk in the forest,

Als it wore a wilde beste (...)

Thare he lifed a grete sesowne;

With rotes and raw venysowne;

He drank of the warm blode (Flowers Braswell (ed.), 1995: 126-127).

El autor de este poema da, pues no solo rasgos animales a Ywain, sino también vampíricos. La animalización de Ywain va a continuar, no obstante, incluso después de recobrar la cordura, ya que sigue sin identidad, sin saber quién es, y llega a decir: "I was a man, now am I nane" (Flowers Braswell (ed.), 1995: 138) y solo se le conocerá desde entonces por el animal de compañía que tiene, un león, por lo que lo llamarán "knight with de lyoun" (Flowers Braswell (ed.), 1995: 152).

\section{LITERATURA ARTÚRICA CONTEMPORÁNEA}

La literatura artúrica contemporánea también ha utilizado animales para crear episodios de características fabulescas dentro de las historias y su parecido con la fábula clásica es más directo que el de los textos medievales. Quizá The Sword in the Stone (1938) es el ejemplo más famoso. Es el primer libro de la pentalogía que T.H. White escribió sobre el rey Arturo y que se completa con The Witch in the Wood (1939), The IllMade Knight (1940), The Candle in the Wind (1958) y The Book of Merlyn (1977, publicada póstumamente). A la pentalogía se la conoce con el nombre de "The Once and Future King", que posteriormente se haría como musical en Broadway (Camelot) así como en una adaptación cinematográfica dirigida por Joshua Logan (1967) e interpretada por Vanessa Redgrave, Richard Harris y Franco Nero. Por su parte, The Sword in the Stone sería la base de la producción de Walt Disney (Merlín el Encantador, 1963). En la obra de White, Merlín entrena al futuro monarca, que todavía no sabe quién es, transformándolo en diferentes animales que, de alguna forma, remedan la Teoría de 
le Evolución de Darwin. Arturo adapta, respectivamente, la forma de un pez, una hormiga, un ganso y un tejón con los que aprende que el poder debe gobernar por el bien de los ciudadanos y que la mejor manera de gobernar es siempre dando libertad. White usa en el texto las palabras Might y Right (poder y bien/derecho) usándolas siempre en mayúscula y, hasta cierto punto, personificándolas.

En el capítulo V, Arturo, al que se llama "The Wart" (que significa "verruga" en inglés y que es un juego de palabras con las primeras letras del nombre), le pide a Merlín que lo convierta en pez en lugar de ir al aula a estudiar. Cuando su profesor le pregunta en qué pez quiere que lo convierta, Arturo responde que en una perca, ya que son "braver than the silly roach, and not quite so slaughterous as the pike are" (White, 1996: 42). Merlín invoca a Neptuno que aparece sentado en una nube con "an anchor tattooed on his stomach and a handsome mermaid with Mabel written under her on his chest" (White, 1996: 42). Inmediatamente Arturo se convierte en perca, pero le pide a Merlín que vaya con él y el mago se convierte a sí mismo en tenca, pero le advierte que en el futuro "you will have to go by yourself. Education is experience, and the essence of experience is self-reliance" (White, 1996: 43).

Mientras están en el foso del castillo, Merlín le enseña a Arturo a nadar y se encuentran con una pequeña pez gobio, cuya madre está enferma y Merlín acude a ayudarla. La madre recupera el apetito, pero Merlín le advierte: “No lob-worm (...) for two days. I shall give you a prescription for a strong broth of algae every two hours, Mrs. Roach. We must build up your strength, you know" (White, 1996: 47). Tras curar a la señora Gobio, Merlín y Arturo se dirigen a ver al señor P (Mr. P), una vieja perca al que llaman el rey del foso y Merlín le pide que le enseñe a Arturo qué significa ser rey. El narrador nos informa que la perca-rey tiene todas las características de un monarca absoluto: crueldad y pena, edad, orgullo, egoísmo y soledad y añade que también tiene una "American expression, like that of Uncle Sam" (White, 1996: 49). En cualquier caso, la perca-rey le comenta que lo que necesita un monarca es, sobre todo, poder: "Power is of the individual mind, but the mind's power is not enough. Power of the body decides everything in the end, and only Might is Right" (White, 1996: 50). Esta última oración, que se ha comentado antes, es ambigua en inglés ya que puede significar tanto que solo el poder es lo correcto, como que solo la fuerza puede y tiene la razón.

Merlín lo convierte a Arturo después en una hormiga, donde aprende a servir a las demás hormigas: es una hormiga-camarero, como se la denomina en el texto. Este episodio también trata de la guerra entre dos grupos de hormigas, tema que luego volverá a tratar para criticar a la raza humana. Las hormigas detectan con sus antenas todo el tiempo la misma canción "Mammy, mammy, mammy", pero hay un momento en el que todas escuchan "Antland, Antland over all", un juego de palabras sobre el título del himno alemán "Deutschland über alles". En su forma de hormiga, Arturo 
también aprenderá que un líder, para sobrevivir, debe matar a otros líderes, tal y como le explica después Merlín: un tipo de hormiga salta sobre la espalda de otra hormiga $y$ "concealed by the smell of her host, she would slowly saw off the latter's head, until she herself had achieved the right of leadership" (White, 1996: 132).

La siguiente transformación en el proceso de aprendizaje de Arturo lo lleva a este a ser un ganso salvaje. Como tal, conoce a Lyó-Liok, una gansa que le enseña la vida en comunidad: cómo se ayudan los gansos y cómo nunca se atacan algo que Arturo no comprende. De los gansos comprende, pues, que hay que ayudarse mutuamente al tiempo que, a través de un cuento que Lyó-Liok le cuenta sobre un ganso escandinavo, aprende sobre la capacidad de un líder para organizar a los demás.

Finalmente, Merlín lo convierte en tejón y le comunica que, como su período de aprendizaje está ya llegando a su fin, esta será la última vez que lo transforme. Cuando se encuentra con el Señor Tejón, que es amigo personal de Merlín, este le comenta que solo le puede enseñar dos cosas: "to dig, and love your home" (White, 1996: 200). No obstante, al final el tejón le lee una parábola que, según él mismo confiesa, “(is) for my doctor's degree, you know" (White, 1996: 202). En esa parábola, dice el señor Tejón, Dios habla con todos los embriones y les da la posibilidad de desarrollarse de diferentes maneras. Solo el último decide quedarse tal y como está y desarrollarse en ser humano, y a él Dios le da el poder "over the Fowls of the Air, and the Beasts of the Earth, and the Fishes of the Sea" (White, 1996: 205), pero el tejón le advierte a Arturo que el final no es tan optimista: "Well, it is true that man has the Order of Dominion and is the mightiest of animals- if you mean the most terrible one- but I have sometimes doubted lately whether he is the most blessed" (White, 1996: 205). Y un poco más adelante, el tejón añade: "Do you know that Homo Sapiens is almost the only animal which wages war?" (White, 1996: 206)

Tras este episodio, a Arturo se lo corona rey, tras sacar la espada del yunque y todos los animales que lo habían ayudado en su formación educativa o bien acuden a la coronación o bien le mandan regalos.

\section{LOS ANIMALES EN SUEÑOS PREMONITORIOS}

Sería conveniente también apuntar brevemente el uso de los animales en sueños como símbolos premonitorios. La literarura artúrica ha hecho buen uso de ellos, desde la lucha de los dos dragones (el rojo, que representa a los británicos, y el blanco de los sajones) que Merlín explica en las leyendas medievales, hasta la visión que tiene Arturo antes de la batalla con el gigante de Mount St. Michel en el barco donde interpretan el sueño en el que él, simbolizado por el dragón, vencerá al gigante, representado por un oso. Este recurso del sueño premonitorio lo han utilizado también autores modernos, 
como Mark Adderley en su The Hawk and the Wolf (2008).

\section{CONCLUSIÓN}

Así pues, podemos ver que la literatura artúrica ha utilizado frecuentemente animales en el desarrollo de sus historias. En el primer libro de la pentalogía de T.H. White hemos visto que los animales tienen una clara función fabulesca de aprendizaje moral, como cuando el tejón advierte a Arturo que la raza humana es de las pocas que ejerce la guerra contra sí misma, mientras que el resto de los animales atacan por defensa o necesidad. Referencias a esto ya aparecieron en la Edad Media, como en el Bestiario de Ramón Llull. El apólogo, en este contexto, en su estructura semiótica, sirve para hacer una crítica velada de la sociedad occidental en particular, creando una serie de microcosmos que aparecen ridiculizados, desde la sociedad marcial de las hormigas, con el que se critica el belicismo de la raza humana, hasta las referencias al Tío Sam en el foso del castillo o la crítica a la guerra que hace el grupo de gansos. No debemos olvidar la fecha en la que T.H. White escribió su texto, justo antes de la Segunda Guerra Mundial, en un período en el que las ideas extremistas, y, muy especialmente, el nazismo y el fascismo, empezaban a ser una amenaza evidente.

En otras historias, los animales son lo que ayudan al héroe a lograr su objetivo, como en los cuentos de los Mabinogion. En su papel como colaboradores, indirectamente también están ayudando al protagonista a mejorar como persona y a instruirse. Además, gracias al auxilio de los animales, la unidad familiar, pilar básico en la sociedad celta en general y en la galesa en particular, queda establecida en la historia. En algunos casos, como hemos visto tangencialmente, los animales también servían para identificar a un héroe con el que creaban una simbiosis, como es el caso del Caballero del León, arriba nombrado.

No nos debe extrañar, pues, que a los animales que consideramos irracionales sean los que critican las costumbres de los que se consideran racionales.

\section{BibliografíA}

Adderley, M. (2008): The Hawk and the Wolf, Marrero, LA., West Bank Publishing.

Browmich, R. y D.S. Evans (eds.) (1992): Culhwch ac Olwen. An Edition and Study of the Oldest Arthurian Tale, Cardiff, Cardiff University Press.

Cuddon, J.A. (1988): Dictionary of Literary Terms and Literary Theory, London, Penguin. Davies, S. (2007): Mabinogion, Oxford, Oxford World's Classics.

Flowers Braswell, M. (ed.) (1995): Ywain and Gawain, Michigan, Western Michigan University.

Knight, S. (1983): Arthurian Literature and Society, London, Palgrave Macmillan.

Lacy, N.J. (ed.) (1997): Text and Intertext in Medieval Arthurian Literature, New York, Garland.

Paxon, J.J. (1994): The Poetics of Personification, Cambridge, Cambridge University Press. 
Reis, C. (2002): Diccionario de Narratología, Salamanca, Almar.

Spang, K. (2000): Géneros literarios, Madrid, Síntesis.

Thorpe, L. (1974): Geoffrey of Monmouth. Historia Regum Britanniae, London, Penguin. "Tres hijuelos había el rey", en P. Úcar Ventura (ed.) (1999): El romancero, Madrid, Magisterio: 105-106.

Von Hendy, A. (2002): The Modern Construction of Myth, Bloomington, Indiana University.

White, T. H. (1996). The Sword in the Stone, London, HarperCollins. 